Anales de Geografía de la Universidad Complutense ISSN: 0211-9803

http://dx.doi.org/10.5209/AGUC.53584

\title{
Turismo rural y paisaje en zonas de montaña. Propuesta metodológica para identificar sus relaciones en las Sierras Meridionales de la Provincia de Albacete ${ }^{1}$
}

\author{
Francisco Cebrián Abellán²; Juan Antonio García González ${ }^{3}$ \\ Recibido: 2 de mayo del 2016 / Enviado a evaluar: 25 de mayo del 2016 / Aceptado: 18 de julio del 2016
}

Resumen. Las zonas de montaña cuentan con especificidades geográficas y socioeconómicas. En ellas, a partir del patrimonio territorial natural y cultural, se ha producido un considerable impulso del turismo rural, que ha centrado el interés en la valoración de la singularidad y variedad de los paisajes. En este caso se presta atención a las sierras meridionales de la provincia de Albacete. El trabajo se apoya en una metodología que combina el uso de encuestas de preferencias de turistas y técnicas cartográficas a partir de algunas variables del sistema turístico en el medio rural. El trabajo se inserta dentro de los paradigmas del desarrollo local y el desarrollo sostenible, aplicado a los espacios rurales y a las zonas de montaña media del sureste de Castilla-La Mancha. La hipótesis se centra en la consideración del paisaje como elemento medular del turismo rural.

Palabras clave: Turismo rural; paisaje; zona de montaña; Albacete; Sierras de Alcaraz y del Segura.

\section{[en] Rural tourism and landscape in mountain. Proposed methodology to identify their relations in the southern midlands in Albacete}

Abstract. Mountainous areas present some geographical and socioeconomic specificities. Within them, it can be found a particular heritage based on natural and cultural landscapes. There, a considerable encouraging of rural tourism, which has been focused on valuation of uniqueness and variety of landscapes, has been developed. In this case, the attention is given to the southern mountains of the province of Albacete, Spain. This work takes its starting point on a methodology that combines the use of surveys showing tourists' preferences, and mapping techniques with variables about touristic rural

1 Este trabajo es resultado del proyecto de investigación: "el paisaje como recurso turístico en el sureste de CastillaLa Mancha: aprovechamiento y puesta en valor". Proyecto anual de investigación científica, financiado por la Junta de Comunidades de Castilla-La Mancha (POII-2014-023-A).

2 Departamento de Geografía y Ordenación del Territorio. Departamento de Humanidades. Universidad de Castilla-La Mancha.

E-mail: francisco.cebrian@uclm.es

3 Departamento de Geografía y Ordenación del Territorio. Departamento de Humanidades. Universidad de CastillaLa Mancha.

E-mail: juanantonio.garcia@uclm.es 
areas. The essay can be located within the paradigms of Local Development and Sustainable Development applied to rural areas and the Midlands in the southeast of Castilla-La Mancha. Our hypothesis focuses on the consideration of the landscape as a key element of rural tourism.

Key words: Rural tourism; landscape; mountain area; Albacete; Sierras de Alcaraz y del Segura.

\section{[fr] Tourisme rural et paysage dans la montagne. Méthodologie proposée pour identifier leurs relations dans le sud Albacete}

Résumé. Les zones de montagne ont des spécificités géographiques et socio-économiques. En eux, du patrimoine territorial naturel et culturel, il y a eu un tourisme rural essor considérable, qui a porté un intérêt sur la valorisation de la spécificité et la diversité des paysages. Dans ce cas, l'attention est payé les montagnes du sud de la province d'Albacete. Ce travail est basé sur une méthodologie qui combine l'utilisation des sondages sur les préférences des touristes et des techniques de cartographie de certaines variables du système touristique dans les zones rurales. Le travail est inséré dans les paradigmes de développement local et le développement durable, appliqué aux zones rurales et les zones de moyenne montagne au sud-est de Castilla-La Mancha. L'hypothèse se concentre sur la prise en compte du paysage comme un élément central du tourisme rural.

Mots clés: Tourisme rural; paysage; région de montagne; Albacete; Sierras de Alcaraz y del Segura.

Cómo citar. Cebrián Abellán, F. y García González, J.A. (2016): Turismo rural y paisaje en zonas de montaña. Propuesta metodológica para identificar sus relaciones en las Sierras Meridionales de la Provincia de Albacete. Anales de Geografia de la Universidad Complutense, 36(2), 237-257.

Sumario. 1. Introducción. 2. La implantación y cambios en el turismo rural en España. 3. El papel del paisaje como recurso turístico. 4. El comportamiento de los turistas y las preferencias en relación al paisaje. 5. Metodologías de análisis. 5.1. Fisiografía. 5.2. Usos y protección del suelo. 5.2.1. Uso del suelo. 5.2.2. Espacios protegidos. 5.3. Infraestructurasturísticas. 5.3.1. Rutas y Miradores. 5.3.2. Alojamientos. 6. Resultados del análisis. 7. Conclusiones. 8. Bibliografía.

\section{Introducción}

El turismo rural ha venido experimentando un crecimiento considerable en algunas comarcas de interior en las dos últimas décadas. La razón hay que asociarla fundamentalmente a los cambios en las preferencias de los turistas, aunque otros factores han actuado en el impulso de esta modalidad, lo que lo ha convertido en instrumento de desarrollo local. Diferentes recursos vinculados a los caracteres del patrimonio territorial y social son los que han actuado como principales motores de atracción de los turistas y de desarrollo del sector. Uno de los ámbitos en los que ha evolucionado más rápidamente es en las sierras meridionales de la provincia de Albacete. En este caso se centra la atención en el sistema turístico de dos comarcas (Sierra de Alcaraz y Campo de Montiel -SACAM- y Sierra del Segura) desde el punto de vista de los recursos paisajísticos y del uso turístico que se hace de estos. El trabajo se apoya en la idea de que el paisaje es un activo territorial fundamental para el turismo rural en las zonas de montaña.

En el artículo se han combinado dos metodologías con objeto de identificar los ámbitos territoriales de mayor uso y valor turístico. Por una parte se ha empleado la 
encuesta a turistas para conocer sus comportamientos y las preferencias, con especial atención a las valoraciones que hacen del paisaje en las sierras meridionales de Albacete. Por otro lado, y a partir de los resultados de la encuesta, se ha empleado una metodología en la que se han seleccionado variables cartográficas relacionadas con el uso turístico del paisaje. La información se ha manejado empleando un Sistema de Información Geográfica. Algunas de las variables se han obtenido de fuentes estadísticas oficiales, mientras que se ha entendido de obligada inclusión y de especial relevancia la elaboración de un inventario de rutas de senderismo de diferente categoría, que tienen una vocación eminentemente turística relacionada con el uso del paisaje. Como resultado, se ha realizado una cartografía de cada una de las variables, y otra de síntesis, que ayuda a identificar los espacios de mayor potencialidad turística y valor paisajístico dentro del territorio.

\section{La implantación y cambios en el turismo rural en España}

El turismo rural es un término de conceptualización compleja. Es difícil de concretar porque no existe una definición compartida y aceptada por la comunidad científica. Esto se debe a la ambigüedad, falta de precisión y claridad de los términos en que se apoya (turismo y espacio rural), al hecho de que existen distintas motivaciones que lo movilizan y porque influyen diferentes elementos en su oferta, que dejan como resultado subtipos dentro de éste (Calls et alt., 1995; Corrales, 1992; Fuentes, 1995; Pulido, 2008; Cebrián, 2008; Vera, 2008). La implantación y evolución del turismo rural en España ha sido relativamente reciente. Desde finales de la década de los ochenta se ha venido produciendo un incremento en el número de viajeros y turistas, a la vez que han crecido los establecimientos de acogida. En estos años, aunque con un cierto retraso, se ha incorporado Castilla-La Mancha y la provincia de Albacete a esta dinámica. Los datos aportados por el INE son ilustrativos a la hora de entender la evolución general de la oferta de alojamientos y plazas a distintas escalas, con un papel destacado de esta Comunidad Autónoma en el contexto nacional. Los primeros datos recogidos por el INE (Instituto Nacional de Estadística: Encuesta de Ocupación de Alojamientos de Turismo Rural, Número de Establecimientos Abiertos por provincias y meses) en el en 2001 dejaban un total de 5.865 alojamientos de turismo rural a escala nacional (de los que 411 estaban en Castilla-La Mancha -lo que suponía el 7\% del total-). En el año 2014 eran 14.682, de los que 1.409 estaban en esta Comunidad Autónoma (9,6\% del total nacional). Durante este periodo (13 años) se ha triplicado la oferta de alojamientos, que se complementan a su vez con otros tipos de establecimientos también presentes en el medio rural, como hoteles o apartamentos en sus diferentes categorías. En el mismo sentido se ha comportado la oferta de plazas, pasando de 44.672 en 2001 a escala nacional (2.937 en Castilla-La Mancha en 2001 6,6\% del total nacional-) a 133.580 en 2014 (12.411 en Castilla-La Mancha -9,3\% del total nacional-) (INE, 2016).

Los cambios han sido también importantes en la demanda, que ha aumentado de manera considerable, ya que durante estos años se ha duplicado el número de turistas en España y se ha triplicado en Castilla-La Mancha (según los datos ofrecidos por el 
INE en 2014 se contabilizaron 174.496 viajeros en esta Comunidad Autónoma -un $6,8 \%$ del total nacional, que ascendía a 2.822.943-), valor que contrasta mucho con el de 2001, que dejaba en esta Región 58.320 en 2014 (4,8\% del nacional, que era en 2001 de 1.210 .889 viajeros). Pero estos indicadores alentadores van acompañados de problemas en el sector. Posiblemente el más ilustrativo es el nivel de ocupación de los establecimientos, que en general es bajo, y que en Castilla-La Mancha se presenta especialmente crítico (la media nacional era de un $15 \%$ en 2014 , mientras que en Castilla-La Mancha no llegaba al 10\%). En este contexto, las sierras meridionales de la provincia de Albacete se han convertido en el territorio que concentra el $30 \%$ de la oferta de establecimientos y de turistas de Castilla-La Mancha, y es el foco de atracción indiscutido de la provincia de Albacete, con más del $80 \%$ de sus establecimientos.

Ha sido por tanto un periodo de crecimiento continuado y de consolidación, aunque con altibajos (entre 2009 y 2012 se ha frenado el crecimiento de la demanda mientras ha seguido aumentado la oferta-, por los efectos de una crisis que se ha ido generalizando en la economía y la sociedad española). Esta modalidad se ha concentrado a escala territorial sobre todo en zonas de montaña; desde el punto de vista del ciclo de vida de los destinos turísticos ha perdido en muchos casos la condición de novedad. Si se considera la demanda, se ha convertido en alternativa para un colectivo creciente de turistas. Y desde el punto de vista socioeconómico de las zonas receptoras ha pasado a tener la condición de instrumento de desarrollo local por algunos de los beneficios que se le han atribuido. Estas razones explican que desde finales de los noventa haya aumentado el interés por el turismo rural. Hay un grupo de autores que ha prestado atención desde disciplinas distintas y enfoques diferentes a los elementos, relaciones, oportunidades, debilidades o conflictos que se han manifestado dentro de su sistema turístico (estructura del sector, actores institucionales, evolución en la oferta, cambios y comportamientos de la demanda, ayudas públicas...) (Bote, 1995; Ivars, 2000; Sancho y Panadero, 2004; Canovés y Villarino, 2005; Pulido, 2008b; Cebrián, 2008; Varela y Martín, 2011; Canovés et alt, 2014; Vera: 2008). Lo cierto es que cuenta con singularidades que lo diferencian de otras modalidades: se organiza a partir de microempresas -muchas de ellas de carácter familiar y sin trabajadores- ; de microdestinos -generalmente de carácter local, ocasionalmente comarcal, y sobre todo en zonas rurales con debilidades estructurales-; y de microproductos - cuando los hay-. Pero, como ya se ha indicado, cuenta con una gran capacidad de crecimiento.

El turismo rural ha venido apostando por estrategias diferentes a las modalidades tradicionales: se apoya en la conservación frente a la depredación (lo que entra en relación con su estructura de pequeños establecimientos, frente a los grandes grupos que dominan otros modelos); en el contacto con el medio (frente al sedentarismo); prioriza la calidad (frente a la cantidad de establecimientos, aunque no siempre se ha conseguido); en la autogestión (frente a la dependencia); en la planificación (que en muchos casos se ha convertido en improvisación); o en el respeto al patrimonio territorial (frente a la transformación generalizada del paisaje) (Reguero, 1994). Descansa en actividades de contacto con la naturaleza, con la cultura local o con el 
patrimonio edificado. Estos principios y caracteres se han reconvertido en estrategias de desarrollo local, que introducen nuevos tejidos productivos; aumentan el empleo, los equipamientos y servicios; incorporan rentas a la población local; diversifican los ingresos de los residentes; conservan el medio... Todo esto convierte al turismo en alternativa y complemento a las rentas y economías tradicionales de las zonas de montaña. Es la búsqueda de la plurifuncionalidad, pero ahora con un componente de innovación y transformación social, ya que el turismo rural ayuda a transferir técnicas de gestión, impulsa la creación de empresas y ambientes propios al desarrollo de actividades turísticas y recreativas, alojamientos o servicios afines. Se suma a todo ello el papel de la planificación integral, con sentido ascendente, desde lo local, con proyectos apoyados en los recursos propios y con una fuerte implicación de la población residente (Ruiz, 2000). Los apoyos institucionales, sobre todo desde las iniciativas Leader (Liasons Entre Activités de Developement de L'Economie Rural) y Proder (Programa Operativo de Desarrollo y Diversificación Económica en Zonas Rurales) financiadas con fondos comunitarios, han sido los principales motores del cambio (Pillet, 2004).

Una de las razones que justifican el crecimiento del turismo rural está en que varían las preferencias de la demanda. Se trata de uno de los factores determinantes en su desarrollo. La clientela está cambiando sus motivaciones y se modifican las razones de los viajes. Los destinos, en sus distintos niveles de desarrollo, están intentando acomodarse y diversificar su oferta ante las nuevas necesidades. Como tónica general se aprecia una sensibilidad creciente por el medio ambiente bien conservado; se asigna un mayor protagonismo al contacto con la naturaleza, la cultura local o con el patrimonio edificado de los espacios rurales; y se incluye una cierta necesidad de movimiento frente al tradicional sedentarismo. Ahora la clientela es más viajera (hipermobilidad en palabras de Urry 2002), con mayor número de desplazamientos que son, por otra parte, de más corta duración y a destinos más cercanos. Estamos ante un turista en busca de experiencias y sensaciones diferentes. En el caso español es sobre todo el mercado nacional el que anima esta modalidad (concentra más del $85 \%$ de los turistas).

Estas transformaciones en la demanda, en la visión institucional y las apuestas empresariales han revalorizado, de forma selectiva, algunos espacios rurales. Pero este proceso ha dejado también sombras en su proceso de implantación. Hay una serie de problemas estructurales, asociados a la estacionalidad, la débil presencia de las empresas de ocio activo, el poco aprovechamiento de las nuevas tecnologías, la escasa cualificación del personal ocupado en el sector, la insuficiente oferta de los puntos de información turística, la precaria señalización, la falta de promoción... (López; 2014: 19). Aparece un modelo reiterado de copia y réplica (Martín y Martín; 2014: 203) fruto de la juventud del sector y de la oferta repetitiva, apoyada en una serie de tópicos como el paisaje, el descanso, el tipismo o la gastronomía tradicional (Varela y Martín; 2011). 


\section{El papel del paisaje como recurso turístico}

El proceso de conformación de los destinos turísticos ha centrado tradicionalmente la atención en el componente más productico y organizativo del sector (empresas, servicios...). Menor ha sido el interés por los recursos, que son la materia prima que lo nutre. Para el caso específico del turismo rural hay un cierto consenso en asumir que es el patrimonio territorial su elemento medular. En especial son los recursos naturales y paisajísticos los que se han convertido en el centro de atención y atracción de los espacios rurales y las zonas de montaña (López.; 2014: 22). La toma de conciencia de esta realidad justifica la tendencia creciente al uso, pero también a la conservación y recuperación. En este contexto de cambio el territorio y el paisaje cobran valor como escenario, como escuela, como aventura, como residencia o simplemente como naturaleza domesticada -sin naturaleza- (Donaire, 2002).

Es preciso considerar que en las áreas de montaña existe un patrimonio territorial, entendido como recurso cultural territorial, y eso supone identificación y reconocimiento social y económico (valor de uso y de cambio), apoyado en la integridad de la herencia social (Ortega; 1998). Esta concepción implica traspasar la visión fragmentada y aislada del territorio para entenderlo como un sistema espacial estructurado, complejo y diversificado. Ha sido elaborado por sociedades en distintos contextos sociales, históricos, económicos, técnicos y políticos, que ha estado y está en proceso de readaptación y cambio permanente. En las zonas de montaña se ha conjugado una tradicional vocación forestal, agraria, ganadera, minera e industrial gestada en modelos socioeconómicos e históricos diferentes. Son por tanto espacios construidos socialmente y dotados de una relativa homogeneidad y diversidad (Ortega; 2004: 6-9). A nosotros nos han llegado algunos en los que conviven coníferas con frondosas (las primeras han ido sustituyendo progresivamente a las segundas en procesos de deforestación-reforestación); usos agrarios de vega en las riberas de los ríos, cultivos aterrazados en las zonas de pendiente más suave y suelos más profundos (hoy en su mayor parte abandonados) o usos arbustivos en los sectores de suelos más ligeros; aprovechamientos ganaderos en zonas de pastos de verano (de los que ahora quedan eriales y apriscos en estado de ruina); explotaciones mineras de localización muy selectiva, y asociados a ellas actividades industriales de diferente naturaleza e impacto territorial (que perviven en la toponimia y en algunos ejemplos de arqueología industrial relacionada con el agua, la madera o el metal, porque la actividad ha quedado reducida, cuando pervive, a museos etnográficos o centros de interpretación). Apenas se mantienen algunos usos ganaderos y agrícolas poco modernizados. Esto explica que las áreas de montaña aparezcan caracterizadas por una despoblación acusada, acompaña de una serie de problemas estructurales asociados a la crisis de sus modelos productivos, que ha derivado en desarticulación económica y social, dejando como balance envejecimiento, o falta de capacitación de sus activos demográficos. De todo ello han resultado los paisajes heredados que comienzan a tener usos diferentes a los tradicionales.

Los paisajes pueden entenderse como conjunto de elementos tangibles, que se convierten en huellas naturales y culturales objetivamente presentes en cada territorio 
y subjetivamente en cada percepción. Esta concepción social y cultural del paisaje se contrapone a la naturalista y fragmentaria, actualmente predominante en palabras de Ortega (Ortega; 2004:13), en la que lo geomorfológico, biológico y ecológico domina. Desde la aprobación del Convenio Europeo del Paisaje (CEP, 2000) han aparecido numerosos trabajos orientados a prestar atención a su conceptualización y a las estrategias de gestión y ordenación (Gómez y Riesgo, 2010; Cortina, 2011; Mata, $2011 \ldots)$. También han proliferado a nivel autonómico instrumentos normativos para dar naturaleza de Ley a lo recogido en el CEP (Cebrián y García, 2016). Pero ahora predomina una visión más integradora, que permite asumir la existencia de un patrimonio territorial singular, propio y diferenciado para las distintas zonas rurales, entre las que se incluyen las de montaña, formado por distintos elementos imbricados que singularizan los territorios, y que los convierten en atractivos. El paisaje asume la condición de activo territorial para funciones asociadas a nuevas demandas de la sociedad (vinculadas con la cultura, el medio ambiente o el turismo). Su importancia está relacionada con los intereses y preferencias de determinados colectivos, que le asignan valor de uso, valor simbólico y valor económico (Ballart y Tresserras; 2005). El paisaje asume, según los planteamientos economicistas del turismo, la condición de objeto de producción de capital, objeto de mercado y por tanto objeto de consumo. En definitiva, el patrimonio territorial se ha incorporado como un insumo mercantilizado que forma parte activa del producto turístico.

Ha habido una convergencia de intereses en el proceso de recuperación del patrimonio de las áreas de montaña como parte de las políticas de desarrollo rural de las Unión Europea desde finales del siglo XX. Se han incorporado estrategias diferentes que le asignan un peso específico considerable en las políticas de desarrollo rural de la UE: por una parte aparecen las de carácter proteccionista; por otro lado están las vinculadas a los paradigmas del desarrollo endógeno y del desarrollo sostenible (Ortega, 2004: 14). Dentro de este contexto el turismo rural ha sido en las zonas de montaña, posiblemente, el principal actor de un anunciado proceso revitalizador (que no siempre ha sido real), en el que el paisaje ha jugado un papel fundamental como elemento emblemático y factor de desarrollo de las áreas de montaña. Esta idea se utiliza como eje discursivo de este trabajo. Las transformaciones sociales operadas en las últimas dos décadas han asignado a los paisajes de montaña la condición de espacios de reserva (en ocasiones sujetos a figuras de protección), con una función de ocio, que se viene multiplicando en la medida en que el tiempo libre se incrementa, el turismo se democratiza, aumenta el número de desplazamientos a destinos más cercanos y se mejoran las infraestructuras de comunicaciones. Estos espacios ofrecen a los habitantes urbanos recursos naturales y culturales (agua, masas forestales, aire, paisajes, tradiciones, cultura local...) que no tienen precio de mercado, pero que cobran un protagonismo creciente en esta modalidad, y se convierte en producto o mercancía cultural (Ojeda; 2004: 274-275). Todo esto hace que la necesidad de adaptación a las necesidades y exigencias cambiantes de nuevos segmentos de demanda despierte la atención por el paisaje como recurso turístico, el paisaje se ha incorporado al proceso de creación de nuevos productos diferenciados, sofisticados, especializados y complejos (Martín y Martín, 
2914: 2014). Aquí entra el deseo de contacto con la naturaleza de manera activa turismo salud, turismo de naturaleza...-.

\section{El comportamiento de los turístas y las preferencias en relación al paisaje}

Uno de los principales problemas es la valoración de los componentes subjetivos del paisaje por parte de los turistas. Habitualmente se emplea el uso de encuestas para entender la importancia que tiene en el sistema turístico. Las realizadas en España sobre preferencias de los turistas dirigidas específicamente al turismo rural son escasas y en muchos casos presentan enfoques diferentes, en función del objetivo y la escala de análisis. A nivel nacional uno de los trabajos pioneros fue el realizado por el Consejo Superior de Investigaciones Científicas (Bote; 1995). Otros han prestado atención a la valoración de imágenes (García, 2010; Mercado, 2015). A escala regional algunas comunidades autónomas, a través de sus observatorios turísticos, han abordado este aspecto mediante encuestas bajo enfoques dispares (en Asturias el SITA, 2013; en Andalucía el SAETA, 2013). A escala de la provincia de Albacete se han elaborado también algunos trabajos que ayudan a entender el papel que desempeña el paisaje en el turismo rural (Cámara de Comercio de Albacete, 2006 y 2008). En todos ellos se resalta el hecho de que es el elemento más significativo en el proceso de elección del destino, y es a la vez el aspecto o elemento más valorado durante la visita.

Por esta razón, y para para entender la función que desempeña el paisaje como recurso turístico en las sierras meridionales de la provincia de Albacete se ha hecho una encuesta a turistas en el verano de 2015 con objeto de identificar los caracteres más relevantes de sus preferencias sobre el paisaje (Cebrián y Sánchez; 2016). Se trata de un estudio piloto, de ámbito comarcal, del que algunos resultados obtenidos permiten hacer una aproximación a los caracteres y las preferencias. Los resultados apuntan a un turista de procedencia nacional, con un nivel educativo medio-alto, que en su mayor parte es trabajador en activo. En su estancia suele tener un comportamiento bastante nómada, ya que recorre diferentes municipios, en viajes con pernoctaciones (de una a tres noches), generalmente en fin de semana o en puente. En cuanto al alojamiento, emplea de forma prioritaria los establecimientos rurales legalizados para uso turístico (casas rurales, hoteles y hostales rurales o albergues), aunque también tiene importancia la segunda residencia o la vivienda de familiares o amigos. La forma de viaje suele ser en familia, en pareja o en grupo. Utilizan el automóvil como principal medio para transporte, y sólo de forma testimonial emplean otros medios (autobús de línea). La selección del destino la realiza directamente sin recurrir a intermediarios (del tipo agencias de viaje). Tiene un cierto conocimiento y fidelidad a la zona, ya que en la mayor parte de los casos ha viajado previamente, y además lo ha hecho con relativa frecuencia. La información la obtiene por tanto mediante experiencia propia o a través de familiares o amigos (lo que lleva a pensar que las oficinas de turismo, las guías, folletos o libros juegan un papel residual en el proceso de difusión de la información y elección del destino). 
Lo realmente significativo es el hecho de que en el apartado de valoración de los recursos turísticos la gran mayoría de los encuestados prioriza lo que se ha identificado como paisaje natural-cultural; también son significativas las respuestas que eligen el patrimonio monumental y los lugares habitados, junto con aspectos como la tranquilidad, la novedad del destino e incluso la accesibilidad. Por detrás quedan los eventos culturales, las fiestas tradicionales y la gastronomía. En situación residual figuran las preferencias por actividades que se realizan en el medio natural o las deportivas, los alojamientos, los servicios o la relación calidad/precio. Se han identificado también aspectos relacionados con la información disponible en señalización, paneles informativos, libros o folletos, Internet o aplicación para móvil, que se entienden como deficientes. En cuanto a los motivos de la elección destacan la visita a espacios naturales y/o protegidos (paisaje natural) y a núcleos rurales (monumentos, museos...). Es significativa la valoración que los encuestados hacen de los recursos, destacando el interés por los paisajes naturales, mientras que el patrimonio cultural (arquitectura, arte, hábitat rural, paisajes cultivados...) queda por debajo, seguido por el patrimonio etnográfico (fiestas, tradiciones, artesanía o gastronomía), las actividades al aire libre o eventos en espacios naturales. La mayoría dan un protagonismo elevado al relieve como elemento simbólico en la identidad del paisaje y también como atractivo turístico. Se lo asignan inmediatamente después a los paisajes del agua.

Destaca el hecho de que se sienten atraídos por actividades de ocio y que, de una u otra forma, participan de ellas. Se decantan por organizarlas y realizarlas por su cuenta, ya que no han encontrado oferta estructurada para la práctica de actividades. Las valoraciones sobre el estado de conservación de los recursos y los entornos son positivas, aunque comienzan a advertir la aparición de elementos impropios que afectan a la calidad del entorno y del paisaje visitado (natural y cultural). Destaca el hecho de que existe una cierta correspondencia entre la imagen previa y la final obtenida una vez concluida la visita turística, con la salvedad de que cuando no hay conocimiento del destino la imagen resultante es mejor a la previa que tenían los encuestados, lo que les anima a regresar. A la vista de los resultados de la encuesta, la cuestión que se plantea es la identificación de los ámbitos territoriales y su valor como escenografías naturales-culturales. En este caso se ha intentado realizar una aproximación, a partir de distintos criterios, a la potencialidad de uso y valor turístico de las unidades de paisaje identificadas en las sierras meridionales de la provincia de Albacete.

\section{Metodología de análisis}

En este trabajo se ha realizado una propuesta metodológica en la que se ha seleccionado un grupo de variables, a las que se ha dado tratamiento mediante un SIG, y que ha permitido generar una serie de mapas temáticos para el ámbito territorial objeto de análisis. El estudio parte de la idea de que la calidad turística del paisaje es mayor donde confluyen determinadas condiciones: presencia de zonas de agua, abundante masa forestal y mayores pendientes (de acuerdo a las preferencias 
manifestadas por los turistas en la encuesta). El otro supuesto parte de la base de que hay mayor aprovechamiento turístico en función de la accesibilidad -rutas y miradores.

La valoración de la potencialidad turística se ha planteado a partir de siete variables (que se han ponderado con una escala ordinal con un rango de uno a cinco). Se han organizado en tres grupos en función de su naturaleza:

a.- Fisiográficas (pendientes e hidrografía). A la vista de los resultados de la encuesta se parte de la idea de que los paisajes más montañosos son más atractivos para el turista.

b.- Uso y protección del suelo (usos del suelo y espacios protegidos). Se parte de la premisa de que los espacios protegidos cuentan con una mayor calidad paisajística.

c.- Turísticas (rutas, miradores y plazas de alojamiento rural). Los sectores afectados por rutas y establecimientos de acogida cuentan con un mayor aprovechamiento turístico.

Figura 1. Modelización del proceso metodológico.

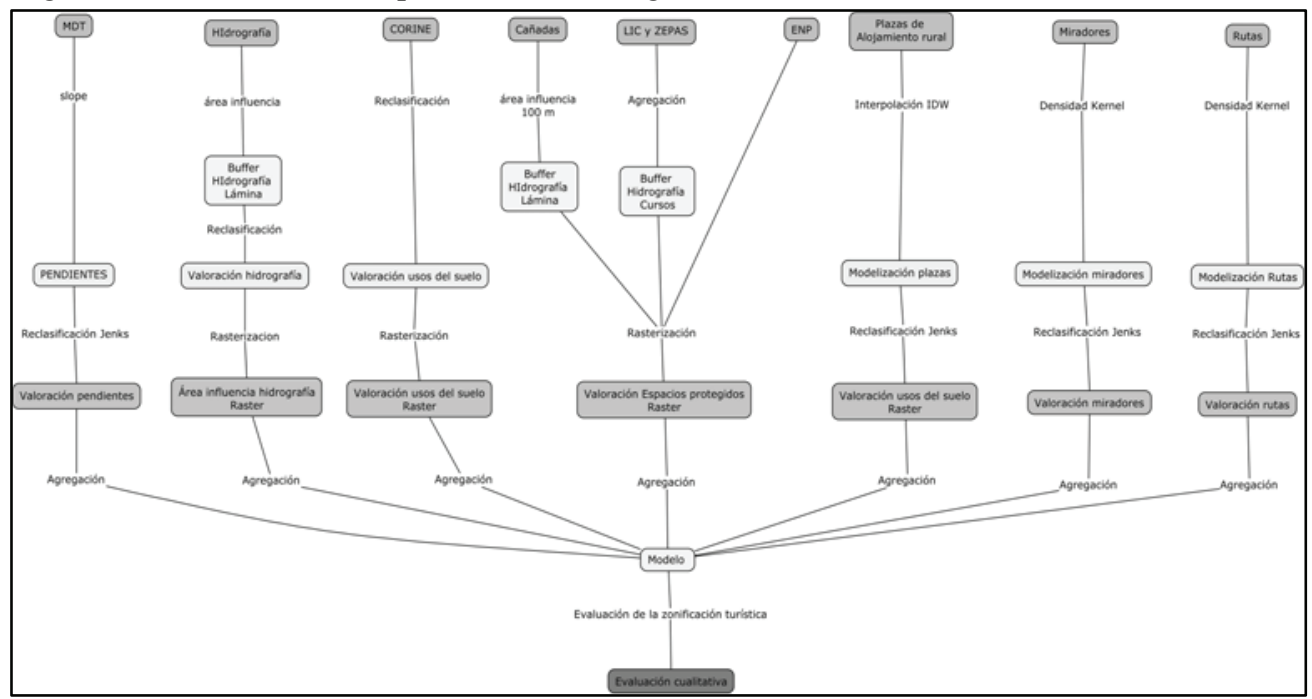

Fuente: Elaboración propia

Las variables se han integrado en un el Sistema de Información Geográfica (ArcGis). Mediante éste se han generado mapas reescalando los valores de cada variable con objeto de homogeneizar y priorizar sus características. A partir de estos valores se ha aplicado una algebra cartográfica agregativa, de la que se ha obtenido un mapa síntesis que permite identificar y establecer una zonificación, diferenciando el potencial turístico de cada unidad en función de las potencialidades y caracteres paisajísticos. Se ha utilizado como cartografía de base la proporcionada por el 
Instituto Geográfico Nacional (IGN) a escala 1:200.000. El proceso metodológico se resume en la Figura 1.

\subsection{Fisiografía}

La orografía es uno de los elementos más significativos en la caracterización de cada unidad de paisaje. Del resultado de la encuesta realizada se desprende que las zonas con mayor valoración son las de montaña y los paisajes del agua. Para cuantificar estos aspectos se ha recurrido a dos variables: las pendientes y la hidrografía.

\subsubsection{Pendiente}

A partir del modelo digital de elevaciones, empleando el curvado del 1:200.000 (I.G.N.), se ha elaborado un mapa de pendientes en celdas de 200x200. Cuenta con un rango que parte de asignar el valor 1 a los sectores llanos, y que alcanza el valor máximo de 5 en los más abruptos (cercanos al 43\% de pendiente). Se trata de una variable continua, que se ha reescalado a partir de la metodología de cortes naturales de Jenks, algoritmo comúnmente empleado en programas de elaboración cartográfica, que minimiza la variabilidad interna de las clases y maximiza las diferencias a partir del número de intervalos que el usuario define, sin dejar valores exactos de los cortes.

\subsubsection{Hidrografía}

La hidrografía se ha entendido como elemento fundamental a la hora de explicar y entender el uso y la valoración turística, visual o recreativa de los paisajes -en este caso del agua- . Se ha empleado la cartografía vectorial 1:200.000 (I.G.N.), que cuenta con una capa de líneas y otra de polígonos que contiene las láminas de agua (naturales y artificiales). El análisis se ha centrado en las proximidades de los cursos fluviales. Se ha optado por un análisis de proximidad a partir de franjas de $100 \mathrm{~m}$, siguiendo las capas de líneas y polígonos que forman la hidrografía. Se ha asignado la máxima puntuación a las zonas más cercanas a los cursos fluviales. Se otorga un valor máximo de cinco a las zonas que se encuentran a menos de $100 \mathrm{~m}$ de los cursos de agua. Esta puntuación se reduce a 3 en sectores ubicados entre 100 y $200 \mathrm{~m}$ de los cursos de agua. A partir de este umbral $(200 \mathrm{~m})$ no se contempla el papel de las zonas hídricas como factor competitivo.

\subsection{Usos y protección del suelo}

En este apartado se incluyen variables que se han considerado representativas a la hora de sintetizar la acción antrópica (usos del suelo) y el valor y singularidad del patrimonio natural (espacios protegidos). 


\subsubsection{Uso del suelo}

El uso del suelo es uno de los aspectos que sintetizan los caracteres del paisaje. En este caso se ha partido de la idea de que las mayores valoraciones turísticas están asociadas a espacios poco antropizados. La capa de usos del suelo recoge las formaciones vegetales y los aprovechamientos agrícolas y de otro tipo. En el entorno analizado la práctica totalidad del suelo ha sido alterado por la mano del hombre, aunque con distintas intensidades y con una considerable superficie de masa forestal heredada. Es a esta última categoría a la que se le otorga un mayor valor.

Se ha empleado la cobertura de usos del suelo de Corine Land Cover (CLC) de 2006. A pesar de la década transcurrida desde la toma de datos se mantienen los caracteres, salvo en algunos núcleos urbanos y en sus periferias (por abandono o por reactivación asociada a segundas residencias turísticas). Se ha hecho una reclasificación de las categorías del tercer nivel de desagregación definiendo cinco tipos. El menor valor corresponde a las zonas urbanas y periurbanas (valor asignado: 1). En el otro extremo (valor asignado: 5) se incluyen las formaciones boscosas de frondosas, coníferas, vegetación esclerófila y los sistemas agroforestales con importantes espacios de vegetación natural. Con valor cuatro aparecen las láminas de agua. Las otras dos categorías hacen referencia a cultivos (valor asignado: 2 herbáceos o leñosos-), y a pastizales y matorrales (valor asignado: 3 ).

\subsubsection{Espacios protegidos}

La variable de los espacios protegidos incorpora al territorio un marchamo de calidad ambiental, que en la mayor parte de los casos refuerza su función turística. Existe un gran abanico de figuras de protección. En el análisis se han tenido en cuenta tres categorías. Por un lado están los que tienen algún tipo de protección europea (ZEPAS -Zonas de Especial Protección de Aves- y LICs -Lugares de Interés Comunitario-). En un segundo grupo aparecen los espacios protegidos que se rigen por la legislación nacional o autonómica (en este caso bajo la condición de Parques Naturales, Reservas Naturales, Monumentos Naturales y Microrreservas). Finalmente, se han recogido en este apartado las vías pecuarias de la comarca (en la actualidad las vías no han asumido la función turística, aunque cuentan con indudables potencialidades por la calidad paisajística de los territorios que atraviesan, en especial la Cañada Real de los Serranos y de Andalucía a Valencia). Para cuantificar esta variable se ha decidido asignar un valor de 5 a las zonas incluidas dentro de un espacio protegido. Se trata en este caso de un mapa de presencia-ausencia, en donde la condición de espacio protegido otorga la máxima puntuación, independiente de la figura.

\subsection{Infraestructuras turísticas}

En este apartado se incorporan algunas dotaciones que participan activamente en el sistema turístico comarcal y en el proceso de disfrute recreativo del 
paisaje. Se han seleccionado por un lado las rutas y la red de miradores; y, por otra parte, se han incluido los alojamientos rurales.

\subsubsection{Rutas y Miradores}

A la hora de entender el uso turístico del paisaje, posiblemente la principal infraestructura al servicio del usuario es la red de senderos y miradores. En la última década se ha producido en las sierras meridionales de la provincia de Albacete un desarrollo considerable de iniciativas orientadas a la puesta en funcionamiento de una red de caminos, senderos y miradores (aunque carente de coordinación). Como sucede en otros territorios son varios los objetivos que mueven a la recuperación de todas estas vías: mantener el uso público de infraestructuras de comunicación; dar respuesta a la demanda social de servicios turísticos alternativos; facilitar a la población el acceso a una experiencia deportiva, cultural, educativa.; favorecer el desarrollo sostenible de la zona... (Porcal, 2011:770). De todas ellas posiblemente la más emblemática por el volumen de inversión y por el efecto territorial es la recuperación de la Vía Verde de Alcaraz (reconvirtiendo en sendero el antiguo ferrocarril Baeza-Utiel, que nunca llegó a entrar en funcionamiento) que recorre casi cien kilómetros desde la capital provincial hasta el límite con la de Jaén, atravesando la Sierra de Alcaraz por uno de los mejores pasos naturales desde la Meseta al Valle del Guadalquivir (Cebrián, 2011). Otra de las iniciativas, que trasciende el ámbito comarcal, pero que ha dejado sus huellas en la Sierra de Alcaraz, es la Ruta del Quijote, creada con motivo de la conmemoración del IV centenario de la publicación de la primera parte del Quijote, y que pese a dejar de tener reconocimiento administrativo desde el año 2012, ha dotado a algunos municipios de una red de senderos que atraviesa algunos de los espacios protegidos o de elevado valor ambiental de la comarca.

A efectos de identificar otras infraestructuras de esta naturaleza, que están presentes, pero que son de menor entidad (porque no están promovidas por instituciones públicas) se ha realizado para este trabajo un inventario de senderos. Se han recopilado los recorridos utilizados y señalizados por entidades locales y/o asociaciones de diversa naturaleza (grupos de desarrollo local, municipios, asociaciones de senderistas...). Se han identificado 77 rutas y senderos repartidos por las comarcas de Sierra de Alcaraz y Campo de Montiel (SACAM) y Sierra del Segura, que por su naturaleza en ocasiones trascienden sus límites geográficos. Algunas tienen la condición de GR o PR, mientras que otras no cuentan con ningún tipo de reconocimiento más allá de la condición de sendero histórico recuperado. Asimismo desde el Grupo de Desarrollo Rural Sierra del Segura se ha creado una red de miradores, en su mayor parte en las carreteras comarcales. Para su homogeneización se han incorporado en una base de datos para su posterior tratamiento en un SIG (coberturas vectoriales para las rutas y otra de puntos para los miradores). En la propuesta metodológica, tanto las rutas como a los miradores se les han asignado la máxima puntuación. A partir de ahí se ha desarrollado un modelo continuo por la interpolación de la distancia inversa, que genera una cubierta donde la valoración del 
territorio disminuye a medida que nos alejamos de la ruta o mirador en cuestión (García y Cebrián, 2004).

Figura 2. Resultados parciales de la valoración de las variables utilizadas en el modelo.

Pendientes

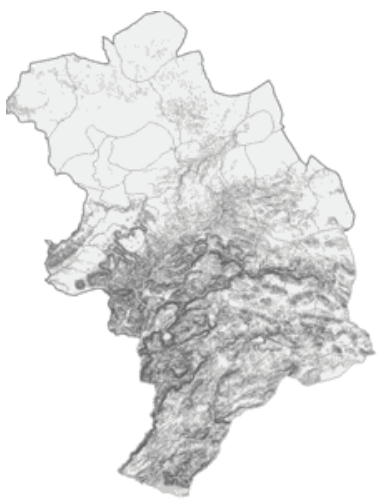

ESPACIOS PROTEgIDOS

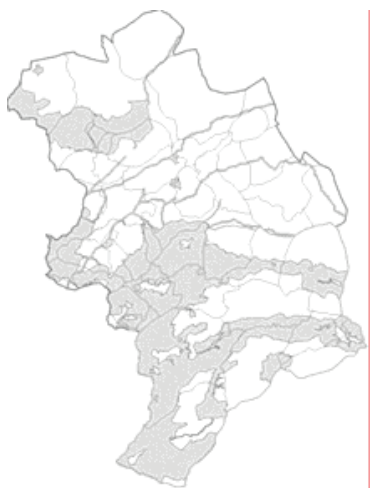

HIDROGRAFÍA

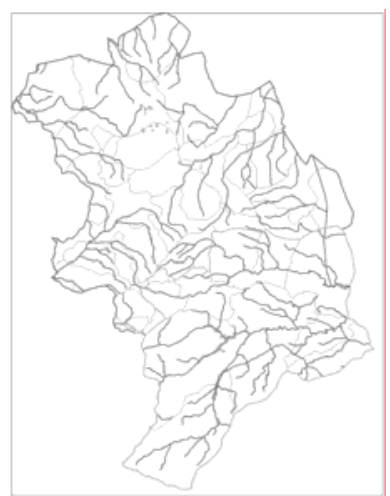

MirADORES

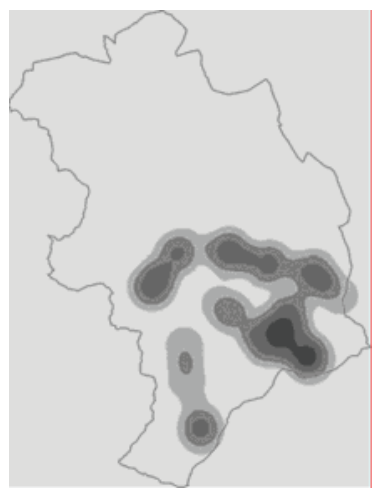

\section{Alojamientos Rurales}

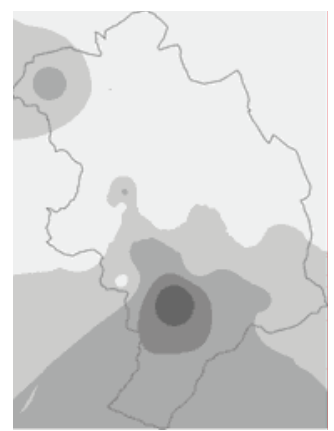

USOS DEL SUELO

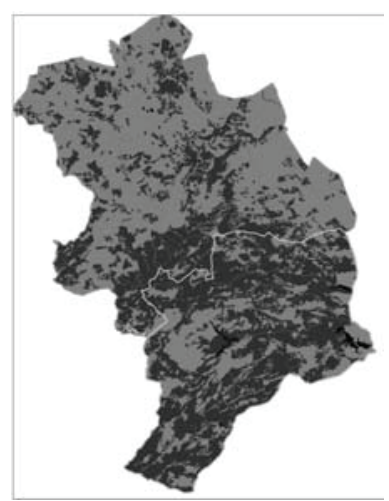

RUTAS DE SENDERISMO

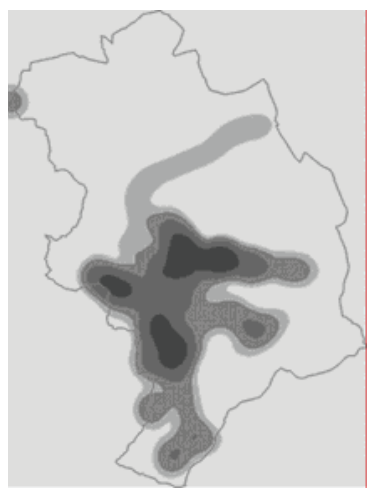

MAPA SíNTESIS

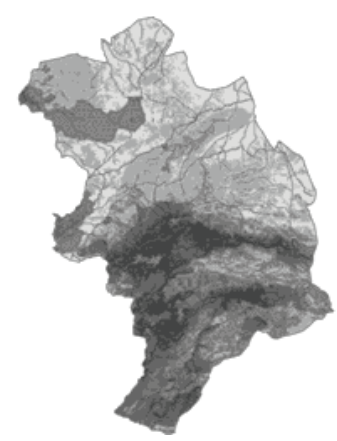

Fuente: Elaboración Propia. 


\subsubsection{Alojamientos}

El alojamiento es otro de los elementos básicos en el proceso de conformación del producto turístico en el medio rural. En este caso se ha empleado la estadística de alojamientos rurales (Dirección General de Turismo, JCCM). El número de plazas de turismo rural en la zona asciende a 3.157 plazas en un total de 594 alojamientos (2015), que están mayoritariamente localizados en el sector meridional (la Sierra del Segura cuenta con 2.219 plazas -donde destacan los casos de Yeste con 808 plazas, Riópar con 254, Letur con 219 y Nerpio con 207- frente a las 938 de la Sierra de Alcaraz y Campo de Montiel -Ossa de Montiel, asociado a las Lagunas de Ruidera y el de Alcaraz con 166 plazas cada uno-). En este caso se ha recurrido para la espacialización de esta variable nuevamente a una interpolación según el método de la distancia inversa. En la Figura 2 se presentan los resultados del análisis en siete mapas, añadiendo también el mapa resultante.

\section{Resultados del análisis}

A partir la propuesta metodológica expuesta se ha generado un mapa de síntesis que combina las siete variables y permite establecer una diferenciación de unidades de paisaje en función de su potencialidad de aprovechamiento turístico. El modelo teórico deja unos umbrales que oscilan entre 5 y 35, siendo 5 el valor mínimo (que es resultado de un punto por la pendiente, uno por el uso del suelo y otros tres por los tres valores de infraestructuras turísticas). En el extremo opuesto aparece la máxima potencialidad turística asociada al valor 35 (la puntuación más elevada se logra a partir de obtener los valores máximos en las siete variables ponderadas) (Figura 2). La Tabla 1 recoge las puntuaciones obtenidas tras los agrupamientos realizados mediante la aplicación del algoritmo de Jenks. En este, una vez aplicada la metodología, se ha obtenido una puntuación que oscila entre 5 y 31 (la tabla incluye también la superficie afectada en cada una de las categorías resultantes).

Estos valores se han representado en la Figura 3 (cinco intervalos según modelo Jenks, de acuerdo a los valores de cada una de las categorías). Se distinguen dos ámbitos territoriales diferenciados. Por un lado está el que recoge los valores más altos (puntuación cinco), y que se localiza junto a los dos grandes cursos fluviales que transitan por el área de estudio. El río Segura y su afluente el río Mundo presentan un doble eje que concentra pendientes y paisajes del agua. Es un sector dominado por calizas jurásicas y cretácicas que ha dejado hoces encajadas con paredes de pendientes acusadas y algunos buenos ejemplos de fenómenos kársticos. Se añade la presencia de importantes masas de coníferas y frondosas. Es en estos entornos naturales de alto valor ecológico donde se concentran las figuras de protección (destaca el Parque Natural de los Calares del Mundo y de la Sima). Esta realidad justifica que se hayan convertido en el ámbito de mayor interés y potencialidad turística, con presencia de alojamientos turísticos, miradores y rutas en las que el paisaje se convierte en el principal atractivo. Es el ámbito de máxima valoración y en el que hay un notable aprovechamiento turístico. 
Tabla 1. Superficie de cada una de los valores resultantes en la ponderación agrupados según las cinco zonas resultantes de la agrupación cartográfica.

\begin{tabular}{|c|r|c|r|}
\hline Puntuación & superficie km & Puntuación & superficie $\mathbf{~ k m}^{\mathbf{2}}$ \\
\hline MUY BAJO & $\mathbf{1 8 7 2 , 5 2}$ & ALTO & $\mathbf{9 0 5 , 8 8}$ \\
\hline 5 & 3,44 & 18 & 219,72 \\
\hline 6 & 1009,52 & 19 & 232,28 \\
\hline 7 & 495,72 & 20 & 238,24 \\
\hline 8 & 363,84 & 21 & 215,64 \\
\hline BAJO & $\mathbf{1 7 5 4 , 9 2}$ & MUY ALTO & $\mathbf{5 4 8 , 1 2}$ \\
\hline 9 & 529,16 & 22 & 187,2 \\
\hline 10 & 394,92 & 23 & 144,52 \\
\hline 11 & 501,48 & 24 & 100,16 \\
\hline 12 & 329,36 & 25 & 59,2 \\
\hline MEDIO & $\mathbf{1 2 2 5 , 5 6}$ & 26 & 30,88 \\
\hline 13 & 259,24 & 27 & 12,32 \\
\hline 14 & 269,44 & 28 & 8,44 \\
\hline 15 & 248,68 & 29 & 3,64 \\
\hline 16 & 229,68 & 30 & 1,56 \\
\hline 17 & 218,52 & 31 & 0,2 \\
\hline
\end{tabular}

Fuente: Elaboración Propia.

Los sectores vinculados a la segunda categoría establecida (valoración cuatro-alto-) se encuentran próximos también a zonas de elevado aprovechamiento turístico y actúan como transición hacia otros de valoración media (puntuación tres), que se pueden entender como sectores con potencialidades de futuro en el proceso de aprovechamiento turístico del paisaje. Con valoración media se incluye el sector de prolongación de las Lagunas de Ruidera hacía el Campo de Montiel y el complejo lagunar en el Bonillo, que podrían actuar como espacios de descongestión de las Lagunas. Por otro lado el Valle del río Guadalmena y especialmente la Sierra del Relumbral (al este de la provincia en el límite con la de Jaén) aparecen también como ámbitos de calidad paisajística media, con indudables potencialidades turísticas. 
Figura 3. Mapa síntesis de la potencialidad turística del paisaje

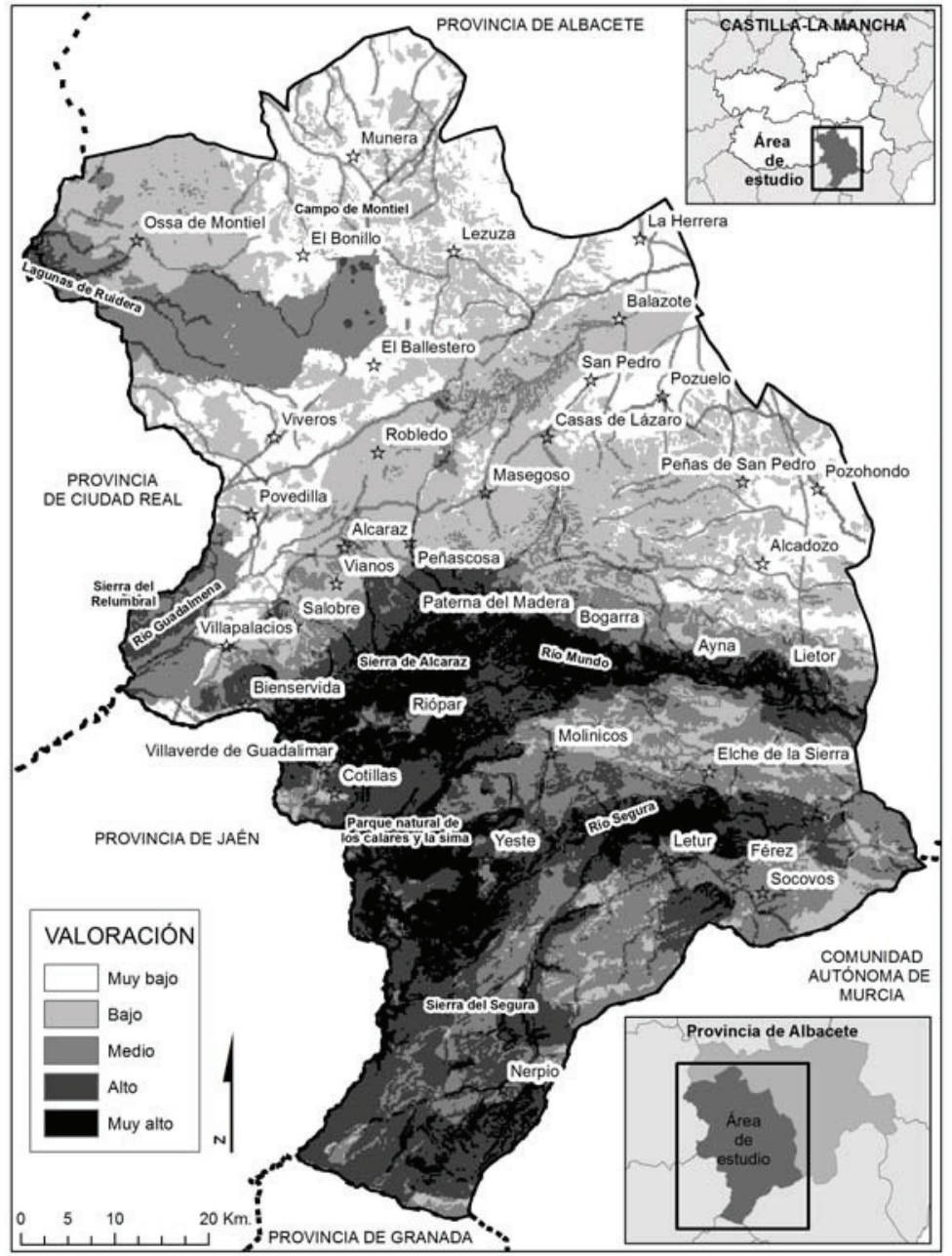

Fuente: Elaboración Propia.

En el otro extremo contamos con las zonas de más baja puntuación (tipo uno y dos - bajo y muy bajo-, con valores que quedan por debajo de la media (16) ocupan más de la mitad de la superficie del área de estudio. Se asume la dificultad de aprovechamiento turístico, aunque podrían combinar el paisaje con otras actividades y modalidades (de hecho así sucede con el caso del turismo cinegético). 


\section{Conclusiones}

El turismo rural en las sierras del sureste de Castilla-La Mancha ha crecido de forma rápida en alojamientos y turistas de procedencia nacional. Se trata de un sector afectado por problemas de diferente naturaleza, entre los que destacan, entre otros, la estacionalidad o los bajos niveles de ocupación. El desarrollo se ha concentrado en ámbitos territoriales en los que se conjuga la presencia de espacios de calidad paisajística con la creciente valoración que de ellos hacen los turistas, que han convertido al paisaje en el elemento de atracción y de preferencia. Entre las propuestas recurrentes para impulsar el sector suele citarse la necesidad de incrementar productos turísticos de carácter comarcal, acompañados de las estrategias de comercialización, de calidad, de mejora de los canales de información o la profesionalización, de dirigir una atención especial a segmentos definidos de la demanda (turistas de retorno, familias, ecoturistas...).

Pero en el conjunto de estrategias suelen tener poca presencia las actuaciones dirigidas al uso y aprovechamiento del recurso fundamental, el paisaje, que es no deslocalizable y posiblemente el más frágil. También se hace preciso considerar la necesaria regulación de actividades y capacidades de carga en aquellos enclaves más sensibles y utilizados con fines de ocio, mientras que otros que cuentan con potencialidades apenas tienen presente esta función, o cuando aparece es casi testimonial. Lo cierto es que el paisaje cuenta todavía con un tratamiento institucional muy débil a escala comarcal, y el interés que hay en su aprovechamiento turístico deja actuaciones voluntaristas y descoordinadas desde diferentes instituciones públicas, sin estrategias decididas a poner en valor su uso recreativo y de ocio. Se hace preciso introducir de manera organizada el discurso del paisaje y sus valores en el colectivo de turistas (y de la población local) que apuesta por el consumo responsable de este recurso.

En este caso la propuesta metodológica permite identifica los ámbitos de mayor potencialidad. Se da la circunstancia de que se perciben desequilibrios acusados, con nodos en los que aparecen fuertes concentraciones de turistas en determinados momentos del año, frente a otros territorios excluidos de los efectos dinamizadores de esta actividad. Es preciso diseñar estrategias correctoras que permitan descargar a los más frágiles desde el punto de vista ambiental y que los beneficios del turismo rural en las zonas de montaña puedan llegar a otros más atrasados en lo social pero con potencialidades. Posiblemente el uso de rutas de senderismo o de redes de miradores puede ayudar a reorganizar y redistribuir el turismo, siempre bajo la premisa de convertir al paisaje en el actor predominante de esta modalidad.

\section{Bibliografía}

Ballart, J y Tresserras, J. (2005): Gestión del patrimonio cultural. Ariel Patrimonio. Barcelona. 
Bote, V. (1995). La demanda turística española en espacio rural o de interior: situación actual y potencial. Instituto de Economía y Geografía del CSIC. Madrid. Documento de trabajo

Cals, J.; Capella, J.; Vaque, E. (1995): El turismo en el desarrollo rural en España. MAPA. Madrid, pp.23.

Cámara de Comercio de Albacete (2006): "Estudio del mercado turístico de la provincia de Albacete". Serie Estudios Turismo, no 1. Cámara de Comercio de Albacete. Albacete.

Cámara de Comercio de Albacete (2008): “Análisis comarcal de la oferta turística de Albacete. Perspectiva de la demanda". Serie Estudios Turismo, n 2. Cámara de Comercio de Albacete. Albacete.

Canovés, G. y Villarino, M. (2005): “Turismo rural en España. Paisajes y usuarios, nuevos usos y nuevas visiones". Cuadernos de Turismo, $\mathrm{n}^{\mathrm{0}}$ 15, pp 63-76.

Canovés, G. et alt. (2014): Turismo de interior: renovarse o morir. Estrategias y productos en Cataluña, Galicia y Murcia. Publications de la Universitat. Valencia.

Cebrián, F. (coord) (2008): Turismo Rural y Desarrollo Local. Universidad de Sevilla y Castilla-La Mancha. Cuenca

Cebrián, F. (2011). "Los ferrocarriles olvidados. Reconversión y reinvención como instrumentos de revitalización recreativa y turística: el Baeza-Utiel a su paso por la provincia de Albacete". Revista Cuadernos de Turismo, $\mathrm{n}^{\circ}$ 27; Universidad de Murcia. Pp. 205-225.

Cebrián, F. (2013) "La función del paisaje como recurso territorial turístico en zonas de interior". Observatorio Medioambiental, vol. 16 37-54

Cebrián, F, y Sánchez, I (2016): “The lanscape as a tourist resource and its impact in mountain áreas in the south of Castilla-La Mancha (Spain)". Sustainable Tourism, 2016. Wessex Institut. Valencia 18-20 de mayo de 2016.

Cebrián, F.; García, C. (2016): "Uso y gestión del paisaje para la actividad turística en el medio rural: aproximación teórica y empírica. Manifestaciones en el SE de Castilla-La Mancha (Albacete)". Boletín de la Asociación de Geógrafos Españoles (BAGE), AGE, Madrid. (en prensa)

Consejo de Europa (2000). Convenio Europeo del Paisaje. Florencia, 20 de octubre de 2000.

Corrales, L. (1992): Apuntes para la definición de turismo rural. Cuadernos de la Escuela Regional de Turismo de Castilla y León.

Cortina, A. (coord.) (2011): El reconocimiento jurídico del paisaje. Articulación del paisaje en el marco normativo español en Gestión del paisaje. Manual de protección, gestión. Barcelona, Ariel.

Donaire, J.A. (2002): "Ecoturismo en zones de montanya: algunes reflexions inicials en Els reptes de lécoturisme en zones de montanya". IV Congres Internacional del medi ambient d'Andorra. Encamp, Centre de Biodiversitat, Institut d'Estudis Andorrans.

Fuentes, R. (1995): El turismo rural en España. Especial referencia al análisis. Madrid, Instituto de Estudios Turísticos.

García, J. (2010): “Ascertaining Landscape Perceptions and Preferences with Pair-wise Photographs: Planning rural tourism in Extremadura, Spain”. Journal of Sustainable Tourism, Pgs. 297-308. 
García, J.A. y Cebrian, F. (2004). "La interpolación como método de representación cartográfica para la distribución de la población: Aplicación a la provincia de Albacete". XII Congreso Nacional de Tecnologías de la información geográfica. Grupo de Tecnologías de la Información Geográfica. Granada.

García, J.A. (2014): Atlas del Turismo residencial. Instituto de Estudios Albacetenses Pp. 214.

Gómez, J. y Riesco, P. (coord) (2010): Marco conceptual y metodológico para los paisajes españoles. Aplicación a tres escalas espaciales. Sevilla, Consejería de Obras Públicas y Vivienda, Centro de Estudios Paisaje y Territorio.

Ivars, J.A. (2000): “Turismo y espacios rurales. Conceptos, filosofías y realidades". Investigaciones Geográficas, $\mathrm{n}^{\circ} 23$, pp. 59-88

López, D. (2014): Los recursos turísticos. Evaluación, ordenación y planificación turística. Estudios de casos. Tirant Humanidades. Colección Crítica. Valencia.

Martín, F.; Martín, I. (2014): “Los espacios rurales españoles ¿territorios donde se produce y consume turismo o destinos sostenibles en entornos competitivos? El caso del noroeste segoviano". Boletín de la AGE, $\mathrm{n}^{\mathrm{0}}$ 64, pp 201-226.

Mata, R. (Coord.) (2008): Atlas de los paisajes de España. Ministerio de Medio Ambiente. Madrid.

Mata, R. (2011): La gestión del paisaje en Retos y perspectivas de la gestión del paisaje en Canarias. Reflexiones en relación con el $10^{\circ}$ aniversario de la firma del Convenio Europeo del Paisaje (Simancas, M. y Cortina, A.). Observatorio del Paisaje de Canarias.

Mercado, I. (2015): "Percepción y valoración social de los paisajes disfrutados: aportaciones desde el visitante para una gestión sostenible de espacios turísticos rurales. El caso de la Sierra de Aracena (Huelva)". Investigaciones Turísticas $\mathrm{N}^{\circ}$ 9, enero-junio 2015, 160-183

Ojeda, J.F (2004): "El paisaje -como patrimonio- factor de desarrollo de las áreas de montaña". Boletín de la $A G E, \mathrm{n}^{\circ} 38$, pp. 273-278

Ortega, J. (1998): "El patrimonio territorial: el territorio como recurso cultural y económico". Ciudades. Revista del Instituto Universitario de Urbanística de la Universidad de Valladolid, $\mathrm{n}^{\mathrm{o}} 4$.

Ortega, J. (2004): “Áreas de montaña: de la supervivencia a la integración”. Boletín de la $A G E ., \mathrm{n}^{\circ} 38$, pp. 5-28.

Pillet, F. (2004): "El desarrollo rural y los instrumentos de la plurifuncionalidad: LEADER y PRODER: las medidas en Castilla-La Mancha en Historia, clima y paisaje". Estudios geográficos en memoria del profesor Antonio López Gómez. Valencia, Universidad de Valencia, UAM y Universidad de Alicante, 539-549.

Pons, B. (Dir) (2011): Atlas de los Paisajes de Castilla-La Mancha. Cuenca, Ediciones de la Universidad de Castilla-La Mancha.

Porcal, M.C. (2011): “El patrimonio rural como recurso turístico. La puesta en valor turístico de las infraestructuras territoriales (rutas y caminos) en las áreas de montaña del País Vasco y de Navarra". Cuadernos de turismo n 27. Pp. 759-784.

Pulido, J.I. (2008): "La investigación del mercado turístico". en Cebrián, F. (edit.): Turismo rural y desarrollo local. Universidad de Castilla-La Mancha y Universidad de Sevilla. Cuenca.

Pulido, J.I. (2008): El turismo rural. Síntesis. Madrid. 
Reguero, M. (1994): Ecoturismo. Nuevas formas de turismo en espacio rural. Madrid, Bosch Turismo, 79-80.

SAETA (2013): Turismo de interior de Andalucía. Informe anual 2013. Junta de Andalucía.

Sancho, J.; Panadero, M. (edit.) (2004). Atlas del Turismo Rural de Castilla-La Mancha. IGN, UAH, UCLM: Madrid

SITA (2013): El turismo en Asturias. Oviedo, 2013.

Urry, J. (2002): “Mobility and proximity”, Sociology, n 36, pp 255-274.

Varela, C.; Martín, F. (2011): "Problemas de sostenibilidad del turismo rural en España". Anales de Geografía de la Universidad Complutense, vol. 31, nº 1, pp. 171-194.

Vera, J.F. (Coord.) (2008): Turismo en espacios rurales y naturales. Atlas Nacional de España. Serie Monografías. IGN. Madrid.

Vera, J.F. (Coord.) (2013): Análisis territorial del turismo y planificación de destinos turísticos. Valencia, Tirant lo Blanch. 